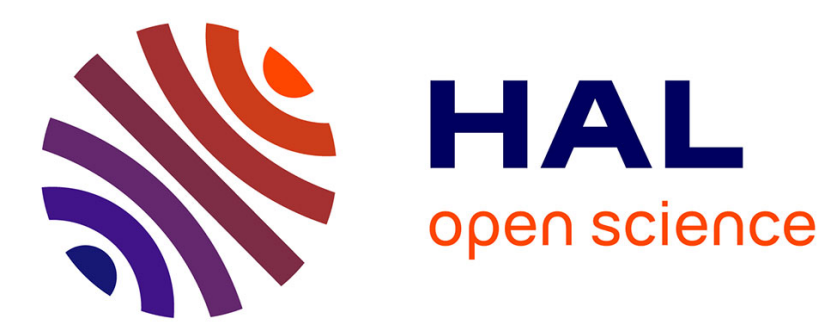

\title{
AUTONOMIE MORALE ET AUTORITE Ou la question de la volonté chez Rousseau
}

Christophe Salvat

\section{To cite this version:}

Christophe Salvat. AUTONOMIE MORALE ET AUTORITE Ou la question de la volonté chez Rousseau. 2009. halshs-00353298

\section{HAL Id: halshs-00353298 \\ https://shs.hal.science/halshs-00353298}

Preprint submitted on 15 Jan 2009

HAL is a multi-disciplinary open access archive for the deposit and dissemination of scientific research documents, whether they are published or not. The documents may come from teaching and research institutions in France or abroad, or from public or private research centers.
L'archive ouverte pluridisciplinaire HAL, est destinée au dépôt et à la diffusion de documents scientifiques de niveau recherche, publiés ou non, émanant des établissements d'enseignement et de recherche français ou étrangers, des laboratoires publics ou privés. 
Groupement de Recherche en Economie $n^{\circ} 2007-14$ Quantitative d'Aix-Marseille - UMR-CNRS 6579 Ecole des Hautes Etudes en Sciences Sociales Universités d'Aix-Marseille II et III

AUTONOMIE MORALE ET AUTORITE Ou la question de la volonté chez Rousseau

\section{Christophe Salvat}

July 2007

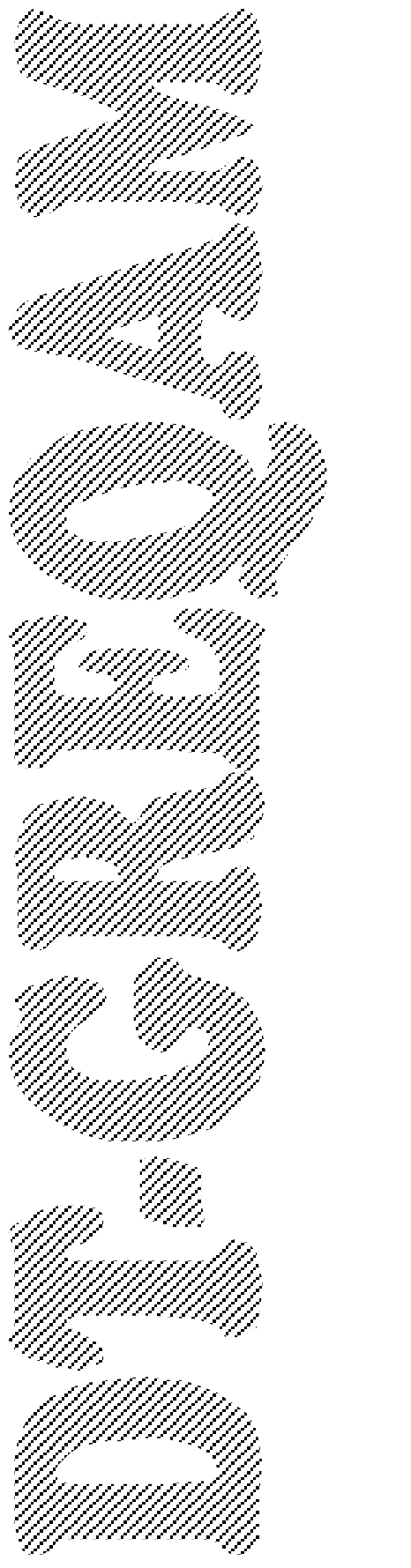




\title{
Autonomie morale et autorité! \\ Ou la question de la volonté chez Rousseau
}

\author{
Christophe Salvat \\ CNRS, Greqam \\ Robinson College \& Centre for History and Economics, Cambridge
}

\section{Résumé}

La notion de souveraineté renvoie généralement dans la tradition rousseauiste à la souveraineté du corps politique et non à celle des individus comme c'est le cas dans les théories du droit naturel. Rousseau, pourtant, ne cède jamais à la tentation holiste. L'individu, et sa protection, reste au fondement de son système. L'objet de cet article est de questionner la notion de liberté entendue comme autonomie morale, en particulier dans sa relation à l'autorité.

\section{Summary}

In the Rousseauist tradition, the idea of sovereignty generally refers to the sovereignty of the body politic. Rousseau never yields nevertheless to the holist temptation. Individual is at the core of his system. The aim of this paper is to question his idea of liberty, understood as moral autonomy, in particular in its relation to the concept of authority.

Classification JEL!: B11!; B31

Mots-Clefs!: Rousseau, Autonomie Morale, Autorité, Liberté, Volonté Générale. 


\section{Introduction}

La notion de souveraineté renvoie généralement dans la tradition rousseauiste à la souveraineté du corps politique et non à celle des individus comme c'est le cas dans les théories du droit naturel ${ }^{1}$. Rousseau, pourtant, ne cède jamais à la tentation holiste. L'individu, et sa protection, reste au fondement de son système. On connaît ainsi les difficultés théoriques auxquelles se confronte Rousseau à l'égard du statut de la propriété individuelle. L'objet de cet article est de questionner la notion de liberté, non du point de vue politique bien connu de tous, mais de celui de l'individu. La notion de volonté individuelle sera notre fil d'Ariane. Elle sera déclinée en trois moments. Dans une première partie, nous mettrons en évidence l'hétéronomie de la volonté et de l'intérêt individuels, et distinguant en particulier les relations d'autorité exercées par la société de la modification des préférences de l'individu face au jugement social. La deuxième partie présente, a contrario, le choix individuel tel qu'on peut se le représenter dans sa forme la plus théorique, autonome et rationnel. Nous nous arrêterons brièvement à cette occasion sur le statut de la fiction dans l'œuvre de Rousseau. Enfin, la troisième et dernière partie s'interrogera sur les conditions de l'autonomie dans l'état civil et sur le rapport conflictuel entretenu entre la volonté individuelle et de la volonté générale. Nous essaierons alors de montrer quelle est la nature de la souveraineté du citoyen.

\section{Intérêt et hétéronomie}

Trois formes de dépendance morale (le terme doit être pris dans son sens large i.e. non physique) décrites par Rousseau conduisent l'individu à agir de lui-même selon un intérêt qui n'est pas à proprement parler le sien, la dépendance morale, la dépendance économique et la dépendance politique. Ces trois types de sujétion de la volonté particulière sont constitutifs de la forme sociale de l'intérêt individuel que Rousseau a désigné par amour-propre. Nous sommes ainsi conduits à distinguer une relation d'autorité qui se traduit par une altération intentionnelle de la volonté d'autrui, qu'elle soit bienveillante ou malveillante, d'une

\footnotetext{
1 Sur le concept de souveraineté chez Rousseau, voir en particulier B. Bernardi, La fabrique des concepts. Recherches sur l'invention conceptuelle chez Rousseau, Paris!: Champion, 2005 et R. Derathé, Jean-Jacques Rousseau et la science politique de son temps, Paris!: Vrin, 1950 (réed. 1995).
} 
adaptation spontanée de la volonté face au jugement social ou à la représentation de l'individu de celui-ci. La première est étroitement associée au statut des inégalités morales dans la société. Plus la société se structure autour d'un rapport à l'inégalité, plus la sujétion de la volonté est nécessaire au maintien de ladite société. Le poids des inégalités morales est renforcé par l'importance accordée au jugement de l'autre dans la construction de l'identité de l'individu. Elle se traduit par une hétéronomie totale de la volonté et l'uniformisation des préférences.

\subsection{Pouvoir et autorité!: la question de la légitimité}

Les relations de pouvoir entre personnes forment la première forme de sujétion de la volonté. Elles représentent a priori la forme la plus visible de la sujétion car elles impliquent généralement un rapport d'autorité fondé sur l'inégalité. L'idée d'autorité associe au rapport de supériorité, déjà présente dans la notion de pouvoir, l'idée d'une obéissance librement consentie à l'exemple de celle du père de famille. Elle implique généralement une inégalité naturelle entre celui qui porte l'autorité (le père de famille, Dieu) et ceux qui lui obéissent (les enfants, les croyants). Dans le cas d'une libre sujétion d'un individu envers son semblable, l'autorité naturelle paternelle ou religieuse est instrumentalisée par ces derniers pour asseoir un pouvoir qu'aucun homme, ainsi que l'a justement noté Hobbes, ne serait capable de conserver par la force. A l'instar de l'opinion publique, ces pseudos relations d'autorité constituent des contraintes, plus ou moins consciemment perçues mais non moins réelles, de la volonté individuelle. Les deux exemples auxquels Rousseau se réfère sont le paternalisme politique et le dogme religieux.

A la différence de l'autorité légitime de la loi, qui exprime la volonté du corps politique, le pouvoir d'un gouvernement illégitime est une autorité travestie. Elle repose sur la croyance selon laquelle le porteur de l'autorité défend l'intérêt de ceux à qui il commande. La question que soulève la libre sujétion à un pouvoir arbitraire est alors la suivante!: Comment parvienton à tromper les individus sur leur propre intérêt!? Le paternalisme politique constitue une forme historique de tromperie, déjà dénoncée par Locke et Sydney notamment ${ }^{2}$, dans laquelle le gouvernant s'octroie abusivement l'autorité paternelle afin d'agir directement sur la volonté

\footnotetext{
${ }^{2}$ Voir à ce sujet l'édition du Discours sur l'économie politique dirigée par Bruno Bernardi, et en particulier le commentaire de F. Guénard «!L’Etat et la famille!».
} 
des gouvernés. L'obéissance ne peut être maintenue durablement par la force. Pour être total, le pouvoir doit apparaître comme le fruit même de la volonté de ceux qui y seront assujettis!: «!l'autorité la plus absolue est celle qui pénètre jusqu'à l'intérieur de l'homme, et ne s'exerce pas moins sur la volonté que sur les actions!» (DEP, OC III, 251). Le consentement au faux pacte par les pauvres dans le second Discours repose sur un dispositif similaire. Son véritable objet, l'institutionnalisation des inégalités économiques, est l'expression d'une volonté particulière, celle des riches à laquelle les pauvres ne peuvent rationnellement se conformer. Dire qu'ils sont libres de se déterminer parce qu'ils sont libres de s'exprimer constitue pour Rousseau une contre-vérité. La véritable dépendance se manifeste, au contraire, lorsque les individus expriment la volonté de ceux à qui ils sont assujettis : «!Tout l'art du maître, écrit-il dans la Nouvelle Héloïse, est de cacher [la] gêne sous le voile du plaisir ou de l'intérêt, en sorte qu'ils [les citoyens] pensent vouloir tout ce qu'on les oblige de faire.!» (NH, OC II, 453). Dans le cas d'une autorité légitime, en revanche, l'individu qui obéit suit toujours son véritable intérêt!; il accomplit une volonté qu'il n'est pas capable d'exprimer, mais qu'il peut (s'il en est capable) rationnellement reconnaître comme sienne. La seule autorité légitime naturelle est celle du père. Elle constituera inévitablement un modèle pour l'autorité politique (CS, OC IIII, 352). A la différence du paternalisme politique, qui désigne une relation entre un gouvernement et le peuple, l'économie politique légitime ${ }^{3}$ caractérise une relation entre le corps politique souverain et les sujets en sont membres. Le principe fondamental du Contrat Social selon lequel «!aucun homme n'a une autorité naturelle sur son semblable!» (CS, OC III, 355) est donc respecté dans le cas de l'autorité politique légitime.

A l'instar de l'autorité politique, Rousseau distingue l'autorité morale de la parole divine, révélée par la Bible, parfaitement légitime, de l'autorité arbitraire, ou plus exactement du pouvoir déguisé, de ceux qui s'en réclament (LEM, OC III, 694). La morale constitue une règle de conduite que l'individu, quelque soit sa religion, se donne à lui-même par la lecture des Ecritures ou l'observation de la nature. Le dogme, en revanche, est l'interprétation de la morale que certains hommes, notamment dans la religion catholique, souhaitent imposer aux croyants. En commandant à ses membres un certain nombre de croyances, dont quelques-unes

\footnotetext{
3 C. Salvat, “Oeconomies. Les articles OE/Economie et leurs désignants dans l'Encyclopédie”, Recherches sur Diderot et l'Encyclopédie, n40, Octobre 2006, pp.97-116.
} 
relèvent certes de la Révélation mais d'autres de la superstition voire de la tromperie ${ }^{4}$, le dogme religieux entretient le culte de l'obéissance et de la crédulité dans l'opinion publique. Ce n'est ni la morale ni la foi des chrétiens que critique Rousseau dans le Contrat Social mais la sujétion qu'elle a instituée. Toutes les religions sont bonnes dès lors qu'elles ne s'octroient aucune autorité sur les hommes ni sur les lois. C'est la raison pour laquelle Rousseau prêche toutes les religions et n'en prêche aucune!à la fois: Wolmar est athée, le vicaire savoyard est catholique, Julie est protestante et Emile embrasse la religion naturelle. Chacun trouve dans sa foi une règle de conduite morale qui lui est personnelle, voire intime, mais qui se concilie parfaitement à la vie civile. Aucune n'est proprement meilleure que l'autre si elle est respectueuse de la libre volonté de chacun. C'est la raison pour laquelle le vicaire savoyard n'est pas prosélyte et le précepteur d'Emile refuse d'aller au-delà de la religion naturelle!: «!Tant qu'on ne donne rien à l'autorité des hommes ni aux préjugés du pays où l'on est né, les seules lumiéres de la raison ne peuvent dans l'institution de la nature nous mener plus loin que la religion naturelle, et c'est à quoi je me borne avec mon Emile. S'il en doit avoir une autre, je n'ai plus en cela le droit d'être son guide!; c'est à lui seul de la choisir!» (Emile, OC IV, 635-36).

\subsection{Hétéronomie de la volonté et opinion publique}

La seconde forme d'hétéronomie de la volonté que nous voulons développer ici n'est ni personnelle ni intentionnelle, elle est sociale. La présence de l'autre, voire la conscience d'une altérité, suffit à modifier nos préférences ou la représentation de notre intérêt. La présence réelle ou virtuelle d'un regard extérieur, tel que le 'spectateur impartial' smithien, n'est jamais légitime car, selon Rousseau, «!Sitôt qu'il faut voir par les yeux des autres il faut vouloir par leurs volontés!» (Emile, OC IV, 309). La prise en considération de l'opinion publique dans le processus de choix de l'individu est doublement dommageable. Tout d'abord, elle contrevient à la liberté naturelle de l'homme, à sa capacité à se gouverner soi-même. L'homme trop sensible au regard et au jugement d'autrui n'est plus souverain!; il est moralement dépendant de ses congénères. A la différence majeure du principe de sympathie, l'opinion n'est ni

\footnotetext{
${ }^{4}$ Voir ainsi la discussion sur les miracles!dans les Lettres de la montagne, ainsi que l'ouvrage qui y est consacré, B. Bernardi, F. Guénard et G. Silvestrini, La religion, la liberté, la justice. Un commentaire des Lettres de la montagne de Jean-Jacques Rousseau, Paris!: Vrin, 2005.
} 
objective ni moralement structurante. Elle conduit, au contraire, les individus à surévaluer les actions destinées à satisfaire leur ego et leur image, au détriment de celles qui leur seraient véritablement utiles et/ou agréables. L'opinion publique représente une forme d'autorité illégitime, bien que diffuse et peu visible, mais dont le rôle institutionnel dans le maintien des inégalités est plus important encore que le gouvernement politique. L'inégalité est, en effet, l'objet même de l'opinion, ce sur quoi se pose le regard du spectateur, et ce sur quoi l'individu espère attirer l'attention. Elle est donc indissociable chez Rousseau de l'idée d'hétéronomie de la volonté. Dans la société civile, l'hétéronomie se construit sur les inégalités morales qui structurent l'identité sociale et la volonté de distinction créée l'uniformisation des préférences qui est constitutive d'une opinion publique.

En l'absence d'un corps politique auquel chacun s'identifie et par lequel chacun est identifié, les individus se construisent une identité sur la différence et la distinction!: «!Chacun commença à regarder les autres et à vouloir être regardé soi-même, et l'estime publique eut un prix. Celui qui chantoit ou dansoit le mieux!; le plus beau, le plus fort, le plus adroit ou le plus éloquent devint le plus consideré, et ce fut là le premier pas vers l'inégalité [...] Sitôt que les hommes eurent commencé à s'apprecier mutuellement et que l'idée de la considération fut formée dans leur esprit, chacun prétendit y avoir droit!; et il ne fut plus possible d'en manquer impunément pour personne» (DOI, OC III, 169-170). L'inégalité, en particulier économique, est donc rapidement perçue comme le support d'une valorisation sociale (Salvat 2007). Elle est, comme l'hétéronomie, consentie par ceux-là mêmes qui en sont victimes, elle «!s'étend sans peine parmi des ames ambitieuses et lâches!» (DOI, OC III, 188). Or, tous les hommes sont victimes des inégalités, car tous sont également dépendants de l'opinion et des préjugés, les maîtres comme les esclaves, les riches comme les pauvres ${ }^{5}$. En plus d'être trompés par les autres, les individus se mentent à eux-mêmes (self-deception) et deviennent dépendants de leurs propres passions. Ils sont, pour reprendre la terminologie de Rousseau, faibles ou, plus exactement, la faiblesse de la volonté est l'une des caractéristiques premières de la nature humaine ${ }^{6}$. L'hétéronomie, et le rôle qu'elle occupe dans le maintien et le développement des inégalités, est certes consentie mais elle n'est pas le produit d'un choix raisonné. Elle est la principale manifestation de la faiblesse de la volonté des hommes.

\footnotetext{
5 «!La domination même est servile quand elle tient à 1'opinion!: car tu dépends des préjugés de ceux que tu gouvernes par les préjugés.!» (Emile, OC IV, 308).

${ }^{6}$ «!C'est la seule tiédeur de nôtre volonté qui fait toute nôtre foiblesse, et l'on est toujours fort pour faire ce qu'on veut fortement!: Volenti nihil difficile.!» (Emile, OC IV, 651). Voir aussi la notion de faiblesse dans l'expression «!Pourquoi l'homme seul est il sujet à devenir imbécile!?!», (DOI, OC III, 142).
} 
Deux exemples nous permettrons de mieux identifier le mécanisme et les effets individuels et sociaux de la sujétion de la volonté individuelle. Le premier, tiré du Discours sur les arts et les sciences publié en 1751, présente la sujétion comme une altération des choix, des goûts et des capacités d'un individu, un artiste ou un savant, destinée à satisfaire les normes et les attentes de l'opinion publique. A moins d'abandonner toute vie sociale, celui-ci n'a en effet d'autre choix que de se conformer à la représentation commune de son art. La liberté dont il peut se prévaloir n'est donc qu'illusoire puisqu'elle implique préalablement une normalisation des préférences. Celle-ci n'est généralement pas consciemment perçue. Elle n'apparaît donc pas contraignante pour une très grande majorité d'intellectuels qui ont intégré les normes artistiques ou académiques avant même de se penser comme artistes ou savants. Elle l'est néanmoins pour les rares individus capables de s'élever au-dessus des représentations communes et qui sont par conséquent conscients de leur dépendance. Deux choix s'offrent alors à ce type d'individu, la sujétion ou l'exclusion. Dans le premier cas, tel Voltaire, il «!rabaissera son genie au niveau de son siecle!», dans le second, il «!mourra dans l'indigence et l'oubli!» (DSA, OC III, 21). Ces cas demeurent cependant exceptionnels. La quasi-totalité des aspirants artistes ou savants, que Rousseau considère comme 'médiocres', a de fait un intérêt réel à adopter ces normes. En masquant leur inaptitude ou leur faiblesse intellectuelle, elles leur permettent de gagner aussi rapidement qu'indûment un statut social élevé. La sujétion des volontés individuelles par l'opinion publique constitue donc une forme de dépendance insensible et quasiment irréversible, propre à accompagner une oppression politique, et que Rousseau compara à «!des guirlandes de fleurs sur les chaînes de fer» (DSA, OC III, 7).

Les normes de consommation constituent une seconde forme de sujétion étudiée par Rousseau. Elles répondent à la fois à la nécessité (ou la faiblesse) morale de se distinguer et à l'impératif économique dicté par la division du travail. Telle qu'elle est décrite dans le Discours sur l'origine et les fondements de l'inégalité parmi les hommes, la division du travail structure le lien social autour des besoins économiques. L'échange réduit l'ensemble des inégalités à une seule, plus visible et plus discriminante, la richesse!: «!Toujours l'objet de l'admiration publique sera celui des vœux des particuliers, et s'il faut être riche pour briller, la passion dominante sera toujours d'être riche!» (CGP, OC III, 964). La conjonction de la volonté de distinction et de la volonté de reconnaissance se traduit par conséquent par une uniformisation des préférences. Ces dernières répondent donc à la fois à une exigence 
économique (satisfaire un besoin ou un désir) et une exigence sociale (se distinguer, se faire admirer). A la différence de la première, la seconde dimension des préférences individuelles implique la prise en compte du regard et des normes de jugement de ses concitoyens. Elle constitue la part hétéronome de l'intérêt responsable selon Rousseau de la dépendance morale des hommes assemblés en société. La configuration la plus radicale de cette dissonance, la consommation somptuaire, constitue aux yeux de Rousseau un modèle d'irrationalité. En accentuant la dimension relative de son utilité, l'individu se contraint à paraître toujours plus riche au prix de son propre bien-être. Les effets de ces comportements se traduisent au niveau économique par une altération du mécanisme de marché et de détermination des prix (les biens les moins utiles et les plus chers sont prioritairement demandés) ainsi que par une redistribution des facteurs de production vers certaines activités manufacturières ou commerciales au détriment des productions de base telle que l'agriculture?

\section{Autonomie morale et rationalité!: rôle des contrefactuels}

L'hétéronomie de la volonté, outre le fait qu'elle questionne un principe de base de la philosophie rousseauiste, celui de la souveraineté, soulève également de nombreuses interrogations sur la rationalité individuelle. L'objet de cette section est d'envisager, d'un pur point de vue théorique, l'autonomie morale de l'individu, c'est-à-dire un état dans lequel il ne serait sujet d'aucune autre autorité que celle de sa propre raison. Cette représentation contrefactuelle de la liberté nous permettra de nous interroger sur les fondements de la notion de volonté, à savoir l'intérêt, ainsi que sur le rôle de la fiction dans l'œuvre de Rousseau.

\subsection{Intérêt et raison}

Les représentations hétéronomes de la volonté et de l'intérêt sont responsables des formes de dépendances exposées précédemment. Elles ne peuvent donc fonder les règles d'un gouvernement libre que ce soit au niveau de l'individu ou a fortiori au niveau du corps politique. Le gouvernement de soi et le gouvernement des autres ont pour principe commun la

\footnotetext{
${ }^{7}$ C. Salvat, «!La loi et l'échange: le statut de la rationalité économique chez Rousseau!», Revue économique, mars 2007,vol.58, n²2, pp.381-398.
} 
prévalence de l'intérêt 'naturel' et rationnel (amour de soi) sur les motivations sociales et passionnées (amour-propre). Le premier est commun à tous les hommes. Son universalité est garantie par sa rationalité. En revanche, en suivant son intérêt tel qu'il peut se le représenter dans le regard des autres (amour-propre), l'individu est en effet condamné à une insatisfaction permanente!: «!L'amour de soi, qui ne regarde qu'à nous, est content quand nos vrais besoins sont satisfaits!; mais l'amour-propre, qui se compare, n'est jamais content et ne sauroit l'être, parce que ce sentiment, en nous préférant aux autres, éxige aussi que les autres nous préférent à eux, ce qui est impossible!» (Emile, OC IV, 493). L'amour de soi est l'intérêt que chacun peut se reconnaître par une stricte raison et une abstraction totale des éléments sociaux propres à fausser le raisonnement. La volonté autonome de l'individu est, par extension, celle qu'il aurait s'il pouvait rationnellement se déterminer au regard de son seul intérêt (amour de soi). La fiction de l'état de nature, ou l'identification à un personnage tel que l'Emile, constituent des expériences de pensée susceptibles de le révéler. Mais, à la différence des autres philosophes des Lumières, Rousseau ne prête au raisonnement qu'une faible capacité à assurer l'intérêt commun et individuel des hommes. La faiblesse de la volonté des hommes, entendez par-là leur incapacité à l'autonomie morale, est la principale cause de leur dépendance. L'autorité politique est donc ce par quoi, dans la société civile, l'homme peut être conduit à respecter son intérêt parfois en dépit de ses inclinaisons!: «!C'est alors seulement que la voix du devoir succédant à l'impulsion physique et le droit à l'appetit, l'homme, qui jusque là n'avoit regardé que lui-même, se voit forcé d'agir sur d'autres principes, et de consulter sa raison avant d'écouter ses penchans.!» (CS, OC III, 364).

Les exemples cités ci-dessus expriment, à des niveaux différents, une seule et même idée sur laquelle repose toute la philosophie politique de Rousseau, à savoir que l'indépendance de la volonté individuelle, parfois associée au concept de liberté, n'existe pas en dehors du strict état de nature. L'homme ne peut pas être libre de ses choix s'il considère l'autre comme spectateur de son action et s'il donne à ce jugement une valeur morale ou sociale. Or, et c'est le principal message du second Discours, la société civile, à la différence de l'état de nature, se structure spontanément autour de hiérarchies fondées sur des inégalités morales (intellectuelles, économiques, politiques et spirituelles). Plus l'organisation de la société est inégalitaire et plus le regard de l'autre est déterminant dans la détermination des choix individuels. La relation inverse entre l'importance accordée aux inégalités dans une société et le degré de liberté de ses membres explique la place accordée par Rousseau à l'opinion publique. En comparant et hiérarchisant les individus selon leurs mérites réels ou supposés, 
elle constitue une véritable caisse de résonance des inégalités sociales et contribue de facto à leur développement ${ }^{8}$. Les lectures les plus critiques de Rousseau voient dans ce processus autoentretenu une impossibilité historique pour l'homme de devenir sujet de sa propre volonté ${ }^{9}$. Nous nous proposons de mettre temporairement cette question de côté pour étudier, dans un premier temps, le rapport de l'autonomie morale à la liberté. Si l'on admet que les formes de dépendance présentées ci-dessus constituent aux yeux de Rousseau une antithèse de la liberté, peut-on conclure a contrario que la liberté se définit par l'indépendance ? La conception rousseauiste de l'état de nature nous fait rejeter cette hypothèse. A la différence des théoriciens du droit naturel, dont il reprend pourtant la méthode et la terminologie, Rousseau distingue en effet nettement l'indépendance et la liberté10 (LEM, OC III, 841).

L'état de nature, au sens strict du terme, i.e. un état hypothétique, un contrefactuel, caractérisé par une indépendance totale de l'homme, ne représente ni la description idéalisée d'un âge d'or ni la conjecture d'un passé probable ${ }^{11}$ mais une référence théorique négative (ou en creux) de la société civil. L'opération intellectuelle a pour but d'identifier la composante sociale du comportement humain. Rousseau virtuellement 'observe' ${ }^{12}$ la nature humaine dans ce contexte totalement désocialisé, puis en déduit la composante acquise. La forte sujétion de la volonté, décrite dans la première section, apparaît mécaniquement comme un effet de la socialisation, mais, et c'est un point fondamental, elle ne signifie pas pour autant que les hommes sont libres dans l'état de nature. L'état de nature rousseauiste soustrait à l'homme toutes ses facultés sociales, dont font partie le langage, la raison et les passions. Seul son instinct le guide dans son action (DOI, OC III, 152). Il n'est donc pas davantage maître de son destin que l'homme civil. L'autonomie, qui s'oppose ici à la faible volonté, se définit, au contraire, comme la capacité de résister aux passions et opinions sociales lorsque celles-ci contreviennent à notre intérêt autonome (amour de soi). La liberté consiste à exercer, par la loi, sa souveraineté en affirmant sa volonté et son intérêt sur ses propres passions. A la

\footnotetext{
${ }^{8}$ !M. Viroli, Jean-Jacques Rousseau and the 'well-ordered society', Cambridge!: Cambridge University Press, 1988; J. Shklar, Men and Citizens. A study of Rousseau's Social Theory, Cambridge University Press, 1969.

${ }^{9}$ Voir en particulier V. Descombes, Le complément de sujet. Enquête sur le fait d'agir de soi-même, Paris!: Gallimard,!2004.

${ }^{10}$ Sur la distinction entre sujet autonome et individu indépendant, et sur ses conséquences sur la représentation de la liberté, voir V. Descombes, Le complément de sujet. Enquête sur le fait d'agir de soi-même, op. cit. pp.332339. Voir aussi J-F. Spitz, La liberté politique, Paris!: PUF, 1995, pp.368-379.

${ }^{11}$ L'erreur des philosophes issus de la tradition du droit naturel, Rousseau pense précisément à Locke, est de n'avoir pas été assez loin dans l'abstraction, et d'avoir construit à rebours un état de nature à partir de l'état civil (DOI, OC III, 132).

${ }^{12}$ L'importance du champs sémantique lié à l'observation dans le second Discours a été encore peu commentée à notre connaissance et est souvent malheureusement trahie par ses traducteurs.
} 
différence de l'indépendance, cependant, l'autonomie morale ${ }^{13}$ est mal définie. Elle n'appartient proprement ni à l'état de nature ni à l'état civil. La fiction qui, à l'instar d'Emile ou de l'éducation, permet de conjuguer les caractéristiques de ces deux états est alors amenée à jouer un rôle prépondérant dans le système rousseauiste.

\subsection{Le statut de la fiction!: Emile}

L'éducation d'Emile, élève explicitement virtuel, est un apprentissage à l'autonomie. Son parcours, de sa naissance à son mariage, a avant tout une portée métaphorique. Emile vit dans un no man's land socialement aseptisé. Le genre discursif de cet imposant ouvrage, entre l'essai et le roman, est volontairement difficile à identifier. Il ne répond que très partiellement aux spécificités de la narration et à celles de la fiction. Emile est le fruit de l'imagination de son précepteur, qui lui est bien réel. Il personnifie en quelque sorte l'homme que Rousseau, dans le second Discours, s'était figuré dans l'état de nature!: «!Nôtre véritable étude est celle de la condition humaine!» précise-t-il dans le livre I (Emile, OC IV, 252). Le choix de la fiction est doublement symbolique. Il traduit, tout d'abord, l'idée que l'imaginaire, à travers notamment l'identification du lecteur à un personnage, peut se révéler être un support de communication plus approprié que l'argumentation ${ }^{14}$. En jouant sur les écarts qu'elle s'autorise à prendre envers son modèle, la fiction véhicule ensuite un propos critique envers la réalité qu'elle conte ${ }^{15}$. L'autonomie morale à laquelle parvient Emile à la fin de l'ouvrage, au moment même où il s'apprête à devenir père, peut ainsi être interprétée comme une critique de la dépendance dans laquelle les hommes sont tombés, et à laquelle ils peuvent échapper individuellement ${ }^{16}$. Mais l'ambition d'Emile est, à notre sens, bien supérieure. Le message délivré ici par Rousseau, et que ses Discours n'ont pas véritablement été capables d'exprimer,

\footnotetext{
${ }^{13}$ Sur le rapport entre autonomie morale et physique (autarcie) chez Rousseau, voir C. Salvat, «!La loi et l'échange: le statut de la rationalité économique chez Rousseau!», Revue économique, mars 2007, vol.58, $\mathrm{n}^{\circ} 2$, pp.381-398.

${ }^{14}$ On retrouve d'ailleurs cette idée dans le Contrat Social, livre II, chapitre VII, dans lequel Rousseau fait reposer l'autorité du législateur sur sa capacité à «!persuader sans convaincre!» (CS, OC III, 383).

${ }^{15}$ La dénomination de roman, explique Rousseau dans le livre V à propos de l'Emile, dépend autant de l'histoire effective des hommes que de celle imaginée de l'un de ses membres!: «!C'est un assés beau roman que celui de la nature humaine. S'il ne se trouve que dans cet écrit, est-ce ma faute!? Ce devroit être l'histoire de mon espèce!: vous qui la dépravez, c'est vous qui faites un roman de mon livre!» (Emile, OC IV, 777).

${ }^{16}$ C'est la thèse notamment soutenue par Robert Derathé. Derathé R., «!L'unité de la pensée de Jean-Jacques Rousseau!», in Baud-Bovy S., Derathé R., Dottrens R. et alii, Jean-Jacques Rousseau, Jean-Jacques Rousseau, Neûchatel!: A La Baconnière, 1962, pp.203-218.
} 
est que l'autonomie morale, cette capacité à se décider rationnellement en-dehors de toute passion sociale, est l'objet même de la loi.

L'autonomie est, tout d'abord, indissociable de l'idée d'autorité. Elle prend, dans le cas d'Emile, deux formes successives, l'autorité de son précepteur, tout d'abord, qui lui apprend à raisonner ${ }^{17}$ et l'autorité qu'Emile doit finalement être capable d'exercer sur lui-même. Comme chaque enfant, il a d'abord besoin de l'assistance d'un parent du fait de son incapacité naturelle et temporaire à être autosuffisant. En l'absence d'une autorité véritable, il transforme cependant rapidement cette dépendance en relation de domination. L'autorité doit alors suppléer à l'assistance pour amener l'enfant à se faire dépendant des choses et non des personnes (Emile 147), et lui donner ainsi les outils de sa future autonomie. Au-delà de l'aspect pédagogique que la question soulève, cette déficience de la raison, présente mais passive, figure d'abord l'incapacité des hommes dans la société civile à se rendre maîtres de leur volonté. Le parcours d'Emile aux côtés de son précepteur ${ }^{18}$, qui du fait de sa fonction a toute autorité sur lui, symbolise le chemin initiatique que les hommes sont appelés à emprunter pour devenir libres. Emile est formé pour vivre parmi les hommes et non en retrait de ceux-ci. Lorsque son éducation prend fin, lorsqu'il s'apprête à devenir père et à rejoindre la société civile, Emile est encore vierge de toute corruption et de toute dépendance!: «!Jusqu'ici, dit le précepteur à son élève, tu n'étois libre qu'en apparence!; tu n'avois que la liberté précaire d'un esclave à qui l'on a rien commandé. Maintenant sois libre en effet!; apprends à devenir ton propre maitre!» (Emile, OC IV, 818). L'autonomie morale, ainsi que nous l'avons déjà mentionné, n'est pas un état libre de toute forme de sujétion ou d'interférence. Elle désigne, au contraire, une situation dans laquelle l'individu est capable, généralement par la

\footnotetext{
${ }^{17}$ Le but de l'éducation est d'apprendre les enfants à raisonner et non de raisonner avec eux («!Le chef-d'œuvre d'une bonne éducation est de faire un homme raisonable, et l'on prétend elever un enfant par la raison!!!», Emile, OC IV, 317). Rousseau reconnaît dans une note du livre II que son vocabulaire peut parfois induire sur ce sujet (comme sur d'autres) en confusion!: «!Tantôt je dis que les enfans sont incapables de raisonement, et tantôt je les fais raisoner avec assés de finesse!; je ne crois pas en cela me contredire dans mes idées, mais je ne puis disconvenir que je ne me contredise souvent dans mes expressions.» (Emile, OC IV, 345).

${ }^{18}$ Rousseau choisit Emile orphelin dans le livre I, mais précise qu'il «!n'importe qu'il ait son père et sa mère. Chargé de leurs devoirs, je succède à tous leurs droits. Il doit honorer ses parens, mais il ne doit obéir qu'à moi!» (Emile, OC IV, 267). Dans le livre V cependant, il légitime le choix de sa future femme, Sophie, sur le transfert d'autorité que le père d'Emile a consenti en lui confiant son éducation!: «!Mon affaire, je dis la mienne et non celle du pére, car en me confiant son fils il me céde sa place, il substitue mon droit au sien!; c'est moi qui suis le vrai pére d'Emile, c'est moi qui l'ai fait homme!» (Emile, OC IV, 765). Un argument similaire est avancé dans le Discours sur l'économie politique. En confiant l'éducation de ses enfants à la nation, le père lui transfère de facto son autorité!: «!Que si l'autorité publique en prenant la place des peres, et en se chargeant de cette importante fonction §l'éducation§, acquiert leurs droits en remplissant leurs devoirs, ils ont d'autant moins sujet de s'en plaindre, qu'à cet égard ils ne font que proprement changer de nom, et qu'ils auront en commun, sous le nom de citoyens, la même autorité sur leurs enfants qu'ils exerçoient séparément sous le nom de peres!» (DEP, OC III, 260).
} 
médiation d'une autorité légitime, d'y résister. Le passage suivant, situé à la fin de l'ouvrage, nous paraît significatif de la portée métaphorique de celui-ci. Après avoir appris à raisonner 'dans le silence des passions', Emile, devenu adulte, reconnaît avoir toujours besoin d'une autorité extérieure pour demeurer maître de lui-même et charge son ancien précepteur d'une nouvelle autorité, la loi, par laquelle il sera capable d'exercer sa propre volonté quelque soient les circonstances, et non de céder à ses faiblesses!:

«!Je ne doute pas un instant que si sur ces maximes j'ai su prendre toutes les précautions nécessaires et tenir à mon Emile les discours convenables à la conjoncture où le progrès des ans l'a fait arriver, il ne vienne de lui-même au point où je veux le conduire, qu'il ne se mette même au point où je veux conduire, qu'il ne me dise avec toute la chaleur de son age, frapé des dangers dont il se voit environné!: O mon ami, mon protecteur, mon maitre!! reprenez l'autorité que vous voulez déposer au moment qu'il m'importe le plus qu'elle vous reste!; vous ne l'aviez jusqu'ici que par ma foiblesse, vous l'aurez maintenant par ma volonté, et elle m'en sera plus sacrée. Défendez-moi de tous les ennemis qui m'assiégent, et surtout de ceux que je porte avec moi et qui me trahissent!; veillez sur vôtre ouvrage, afin qu'il demeure digne de vous. Je veux obéir à vos loix, je le veux toujours, c'est ma volonté constante!; si jamais je vous desobéis ce sera malgré moi!; rendez-moi libre en me protégeant contre mes passions qui me font violence!; empêchez-moi d'être leur esclave et forcez-moi d'être mon propre maitre en n'obéissant point à mes sens, mais à ma raison.!» Emile, 487-88, folio.

Emile, la Nouvelle Héloïse ${ }^{19}$, ainsi que d'une certaine manière les écrits autobiographiques de Rousseau $^{20}$, expriment une même idée, à savoir que l'autonomie morale est indissociable de la notion d'autorité. Dans le cas d'Emile et de Saint-Preux, elle est personnifiée par un homme de raison, maître de lui-même et de ses passions, et capable de guider son élève ou

\footnotetext{
${ }^{19}$ La question de l'autonomie morale, et de son apprentissage, prend une nouvelle forme dans la relation de Wolmar et de Saint-Preux. L'ambiguïté du caractère fictionnel de Julie ou la Nouvelle Hélö̈se, quoique plus affirmé que celui d'Emile, est également savamment entretenue par sa forme épistolaire ainsi que sa présentation éditoriale. Le roman s'ouvre sur une situation de dépendance morale, celle de Saint-Preux qui, follement épris de Julie, lui envoie trois lettres successives pour lui signifier son malheur, et la supplier d'y mettre un terme. Il se poursuit comme un apprentissage à la raison et à la maîtrise de soi. Les voyages et l'autorité d'un Mentor constituent les deux éléments essentiels du chemin initiatique de Saint-Preux qui, tel Télémaque, parvient finalement à se rendre maître de ses passions. Wolmar en est, avec Milord Edouard, l'élément clef. Les figures de l'autorité dans la Nouvelle Héloïse ont été analysées avec une grande intelligence par Judith Shklar. J. Shklar, Men and Citizens. A study of Rousseau's Social Theory, Cambridge University Press, 1969.

${ }^{20}$ Une étude similaire pourrait être menée à partir de l'expérience même de Rousseau et de ses écrits autobiographiques. Nous avons choisi de ne pas traiter la question ici. Nous renvoyons aux travaux de Starobinski, et notamment J. Starobinski, Jean-Jacques Rousseau : La transparence et l'obstacle, Paris!: Gallimard, 1971.
} 
son ami selon les lumières de la raison. Dans les deux cas, cependant, comme dans le cas de l'éducation d'un enfant, cette tierce autorité n'est que transitoire. L'autonomie n'est jamais assurée, on est toujours tenté par ses passions, la loi prend en quelque sorte le relais de l'éducation.

3. Autorité politique et autonomie morale

Dans le contexte politique de l'état civil, la liberté (ou la non sujétion de la volonté) ne peut prendre la forme du retrait ou de l'isolement intellectuel de l'économie domestique de Clarens ou de l'éducation domestique d'Emile. Est libre, dans la tradition républicaine ${ }^{21}$, celui qui sait rester maître de lui-même face aux aléas du hasard, de la fortuna ${ }^{22}$, et non celui qui fuit les sources de nuisance. Le citoyen libre est celui qui, dans le même temps, a une totale autorité sur lui-même et qui obéit aux lois!. Cet apparent paradoxe est, chacun le sait, l'objet même du Contrat Social. Au regard de ce qui a été traité dans la première partie, la difficulté à laquelle se confronte Rousseau concerne la nature et la légitimité de l'autorité de la loi. Elle ne peut être facteur de sujétion, de contrainte ou d'hétéronomie. Nous montrerons donc, dans un premier temps, que le concept de volonté générale est ce par quoi l'individu exerce une autorité sur soi, et que, c'est dans le conflit que cela génère qu'il exerce vraiment sa souveraineté.

\subsection{Avoir autorité sur soi}

La nature de la loi, lorsque celle-ci est légitime, est tout d'abord de ne pas être source d'hétéronomie. Dans le cas contraire, elle ne peut être garante de la liberté des individus et manque par conséquent à son principal objet. La loi est une forme d'autorité qui,

\footnotetext{
${ }^{21}$ Philip Pettit et Quentin Skinner ont largement contribué ces dernières années à défendre le concept de liberté républicaine, entendu comme une non domination (par opposition à la non coercition de la liberté négative). P. Pettit, Républicanisme. Une théorie de la liberté et du gouvernement, Paris!: Gallimard, 2004. Voir aussi Q. Skinner, «!Un troisième concept de liberté au-delà d'Isaiah Berlin et du libéralisme anglais!», in Actuel Marx, $\mathrm{n}^{\circ} 32$, Les libéralismes au regard de l'histoire, 2002, pp.15-49.

22 J. G. A. Pocock, Le moment machiavélien. La pensée politique florentine et la tradition républicaine atlantique , Paris!: Presses Universitaires de France, Léviathan, 1997.
} 
contrairement aux dites autorités décrites dans la première partie, ne peut influencer ou assujettir les volontés individuelles. Sa nature est définie dans les termes mêmes du contrat social que Rousseau formule ainsi!: «!Trouver une forme d'association qui défende et protege de toute la force commune la personne et les biens de chaque associé, et par laquelle chacun s'unissant à tous n'obéisse pourtant qu'à lui-même et reste aussi libre qu'auparavant!»(CS, OC III, 360). La forme singulière choisie par Rousseau ('chacun') pour désigner la pluralité des individus est significative. Pour que la loi exprime toutes les volontés particulières, elles doivent logiquement toutes s'identifier à une seule, la volonté générale. Loin de signifier que tous les hommes sont identiques et motivés par un même intérêt, cette formule rend simplement compte de la nature commune et rationnelle de la volonté générale.

La volonté générale est la volonté, non des individus réunis, mais du tout constitué en corps moral!: «!A l'instant, au lieu de la personne particuliere de chaque contractant, cet acte d'association produit un corps moral et collectif composé d'autant de membres que l'assemblée a de voix, lequel reçoit de ce même acte son unité, son moi commun, sa vie et sa volonté!» (CS, OC III, 361). La métaphore organique du corps politique, bien que Rousseau lui reconnaisse par ailleurs une pertinence limitée $e^{23}$, lui permet de construire dans un premier temps une représentation rationnelle et autonome de la volonté. Celle-ci «!est toujours droite et tend toujours à l'utilité publique!» (CS, OC III, 371). Le corps politique, à la différence de ses membres, se détermine en fonction d'un intérêt unique et commun à tous. Il est l'intérêt que chacun peut rationnellement et objectivement reconnaître comme sien, celui que l'état de nature a permis d'identifier comme celui de chaque homme (amour de soi), mais dont la faiblesse de la volonté prévient en partie l'accomplissement. Par sa nature même, le corps politique est donc souverain, c'est-à-dire qu'il est capable d'exercer sa libre volonté. Il représente aux yeux de tous l'idée même de la force (CS, OC III, 360). Mais il n'est qu'une représentation. Le pacte social qui le produit n'est pas un accord entre les individus qui le composent, mais un accord entre soi et l'idée que nous nous faisons du tout. Il s'énonce ainsi!: «!Chacun de nous met en commun sa personne et toute sa puissance sous la suprême direction de la volonté générale; et nous recevons en corps chaque membre comme partie indivisible du tout. » (CS, OC III, 361). Le corps politique n'a d'existence que celle que lui

\footnotetext{
${ }^{23}$ Voir la métaphore du DEP «!Qu'on me permette d'employer pour un moment une comparaison commune et peu exacte à bien des égards, mais propre à me mieux faire entendre. Le corps politique, pris individuellement, peut être considéré comme un corps organisé, vivant, et semblable à celui de l'homme!» DEP, OC III, 244. Cf R. Derathé, Jean-Jacques Rousseau et la science politique de son temps, Paris!: Vrin, 1950 (réed. 1995), pp.410413.
} 
reconnaît l'individu. Il prend donc logiquement forme au moment même où l'individu passe un contrat moral avec lui, au moment même où l'individu se reconnaît comme une partie indivisible de celui-ci. Le contrat social associe donc deux idées, celle d'identité et celle d'autorité. Il se présente d'abord comme une modification par l'individu de sa propre perception parmi les autres. Celui-ci se percevait auparavant comme un moi absolu -un individu-, il se perçoit dorénavant comme un moi commun -un citoyen- (Emile, OC IV, 249). Mais, et c'est en cela que le contrat lui est nécessaire, il institue une autorité sur soi-même. Ces deux aspects sont, ainsi qu'on va le voir, indissociables dans la pensée de Rousseau.

Le contrat rousseauiste est, aux dires mêmes de son auteur, particulier dans le fait qu'il ne reconnaît pas la notion d'altérité qui fonde traditionnellement l'idée d'obligation. Chaque individu contracte 'pour ainsi dire' avec lui-même (CS, OC III, 362). Le contrat est donc un artefact par lequel l'individu s'oblige lui-même. Il se donne en quelque sorte autorité sur luimême par la médiation de la loi. L'objet de cette convention est, à notre sens, de se donner les moyens d'exercer sa volonté. La faiblesse naturelle de la volonté des hommes les a historiquement conduit vers un état de dépendance et d'inégalité, contraire à l'intérêt de chacun, et qu'aucun homme ne peut rationnellement et objectivement choisir. La nature de la loi est de protéger les hommes, et donc, avant tout, de les protéger contre eux-mêmes. Elle oblige les individus «!à conformer leurs volontés à leur raison!» (CS, OC III, 380). La loi ne peut cependant être comparée à une résolution personnelle, un contrat moral que l'on passe avec soi-même, elle tire son autorité de la reconnaissance par l'individu du corps politique et du statut de la citoyenneté qui l'accompagne. En se pensant comme partie d'une communauté, dont tous les membres partagent le même intérêt, l'individu reconnaît dans la règle commune une manière de se conformer à sa propre volonté. Elle «!lui apprend à agir selon les maximes de son propre jugement, et à n'être pas en contradiction avec lui-même!» (DEP, OC III, 248).

En tant que membres du corps politique souverain, les individus sont libres, c'est-à-dire capables de se gouverner de façon autonome et rationnelle. Les citoyens exercent leur souveraineté par la médiation de la loi. En tant que sujets, cependant, ils sont soumis à une autorité qui contrevient nécessairement à leurs volontés et désirs immédiats!: «!L’essence de la souveraineté consistant dans la volonté générale, on ne voit point non plus comment on peut s'assurer qu'une volonté particuliére sera toujours d'accord avec cette volonté générale. On doit bien plustôt présumer qu'elle y sera souvent contraire!; car l'intérest privé tend toujours aux préférences et l'intérest public à l'égalité!» (Emile, OC IV, 843). Il y a de l'aveu 
même de Rousseau un conflit permanent entre volonté particulière et volonté générale, ou entre intérêt particulier et intérêt commun. L'homme préfère en effet toujours son intérêt à celui de la communauté à laquelle il appartient, et plus la communauté est large plus sa préférence sera accentuée, «!En sorte, écrit Rousseau, que chacun est prémièrement soimême, et puis magistrat, et puis citoyen. Gradation directement opposée à celle qu'éxige l'ordre social.!» (Emile, OC IV, 845). Si la loi rend l'homme libre en lui donnant les moyens d'exercer sa souveraineté, d'agir selon sa volonté autonome, peut-elle le contraindre sans briser la condition d'autonomie, sans devenir elle-même une source d'hétéronomie!? Si tel est le cas, quel moyen a l'individu de reconnaître une autorité usurpée d'une autorité légitime!? Si la nature de la loi est de forcer l'individu à préférer l'intérêt commun à son intérêt particulier, alors les interprétations faisant de Rousseau une source de légitimité du despotisme sont, croyons nous, irréfutables. L'ambition de notre dernière section est de montrer cependant que ce n'est pas le cas.

\subsection{La condition de la liberté}

La présence de la loi se concrétise immédiatement pour l'individu par une distance perçue entre sa volonté particulière et la volonté générale. Elle joue donc d'abord un rôle de signalisation. En revendiquant sa légitimité, à savoir le fait, avéré ou non, qu'elle exprime la volonté générale, elle signale la nature de cette distance. L’individu connaît dès lors son intérêt 'objectif'. Il est donc en état de délibérer, à chaque fois qu'une divergence se présente, entre ce que la pression sociale ou ses passions lui dictent, et ce que la raison publique lui prescrit. S'il choisit la première option, il cède à l'hétéronomie!et à l'amour-propre; s'il prend la seconde, il choisit l'autonomie et l'amour de soi. Cette action délibérée, faite volontairement sans contrainte ni menace, et répétée à chaque fois que le cas se présente, permet donc à l'individu d'exercer, par la médiation de la loi, une autorité sur lui-même et par conséquent de vaincre la faiblesse de sa volonté.

La distinction entre amour de soi et amour-propre, entre une représentation autonome et hétéronome de l'intérêt particulier, n'est pas historiquement fondée. Pas plus que ne l'est d'ailleurs l'état de nature. Elle établit une distance entre un fait, les hommes sont passionnés et se déterminent comme tels, et un référent, celui de la raison. Malgré la forme faussement 
narrative adoptée dans le Discours sur les inégalités, il n'y a donc pas d'évolution ou de transformation de l'amour de soi en amour-propre, et l'on ne saurait voir a posteriori dans l'institution d'un état civil un 'retour' à l'amour de soi. Les volontés particulières et la perception de l'intérêt individuel restent évidemment hétéronomes. L'autonomie morale d'Emile est non seulement fictive mais virtuelle. Rousseau n'attend d'aucun homme une telle perfection. La réflexion politique repose, au contraire, sur les moyens conventionnels de pallier aux imperfections naturelles de l'homme ainsi que le rappelle la première phrase du Contrat social ${ }^{24}$. La divergence entre volonté particulière et volonté générale, évoquée cidessus, reflète donc simplement la distance qu'il existe entre l'amour de soi et l'amourpropre. En exprimant la volonté générale, la loi donne à voir l'intérêt 'naturel' et rationnel partagé par tous. Elle implique l'idée de délibération, de choix voire de conflit entre ce vers quoi nos passions nous conduisent spontanément et ce vers quoi notre raison devrait nous conduire. L'homme raisonnable est donc constamment partagé entre la satisfaction de son intérêt socialisé (amour-propre) et celui de son intérêt 'naturel' ou rationnel (amour de soi). La nature de la souveraineté réside dans l'exercice de ce choix.

Le point sur lequel nous voulons insister ici, c'est que, loin d'être un état en soi, celui de la sécurité ou de l'absence d'interférence, la liberté se manifeste pour Rousseau dans l'exercice répété de la souveraineté, dans la capacité de l'individu à vaincre la faiblesse de sa volonté. L'état civil légitime garantit certes la liberté politique (la souveraineté du corps politique) mais la liberté civile, c'est-à-dire la souveraineté de l'individu, n'est quant à elle jamais garantie. Elle ne peut l'être, en tout état de cause, lorsque l'individu se croit durablement protégé de toute forme de dépendance ${ }^{25}$. La liberté, au sens républicain du terme, se révèle au contraire dans l'épreuve, dans la capacité du sujet à maîtriser la fortuna : « il n’y a point de bonheur sans courage ni de vertu sans combat. Le mot de vertu vient de force ; la force est la base de toute vertu. La vertu n'appartient qu'à un être foible par sa nature et fort par sa volonté (...). Qu'est-ce donc que l'homme vertueux!? C'est celui qui sait vaincre ses affections. Car alors il suit sa raison, sa conscience, il fait son devoir, il se tient dans l'ordre et rien ne peut l'écarter. » (Emile, OC IV, 817-18). A l'instar des philosophes républicains,

\footnotetext{
24 «!Je veux chercher si dans l'ordre civil il peut y avoir quelque regle d'administration légitime et sûre, en prenant les hommes tels qu'ils sont, et les loix telles qu'elles peuvent être.!» (CS, OC III, 351)

${ }^{25}$ «!Plus j'éxamine l'ouvrage des hommes dans leurs institutions, écrit Rousseau dans l'Emile, plus je vois qu'à force de vouloir être indépendans ils se font esclaves, et qu'ils usent leur liberté-même en vains efforts pour l'assurer. (...) Il me semble que pour se rendre libre on n'a rien à faire!; il suffit de ne pas vouloir cesser de l'être.» (Emile, OC IV, 855-56).
} 
Rousseau présente l'autodétermination à la liberté comme une qualité morale, la force de la volonté ou vertu, nécessaire à la citoyenneté (DEP, OC III, 251). Celle-ci ne désigne pas une obéissance absolue et aveugle à la loi mais la reconnaissance de la part de l'individu de sa faiblesse naturelle et de son besoin, pour se rendre libre, de la médiation d'une autorité légitime, celle de la loi. La liberté repose sur un équilibre extrêmement fragile, sur un rapport de force toujours incertain entre les volontés particulières et la volonté générale. Le corps politique, explique Rousseau, est destiné à s'autodétruire à plus ou moins longue échéance, il «!porte en lui-même les causes de sa destruction!» (CS, OC III, 424). L'objet de la réflexion politique, et de la sienne en particulier, est alors de prolonger cette échéance autant que possible en étant conscient, et en faisant prendre conscience, de la nature humaine et de l'imperfection de toute convention. La souveraineté du corps politique, pas plus que celle de ses membres, n'est jamais acquise. Elle doit, elle aussi, faire l'objet d'une volonté forte et permanente.

\section{Conclusion}

La notion d'autonomie morale que nous avons introduit ici pour aborder la question de la liberté répondait à une source d'insatisfaction. Les distinctions usuelles de liberté positive et négative, ainsi qu'à certains égards leur critique républicaine, nous semblaient particulièrement inappropriées dans le cas de Rousseau dans la mesure où elles réduisent, après Constant, l'indépendance et la souveraineté à la sécurité et la participation citoyenne. Notre ambition a été de montrer combien la question de la volonté individuelle, notamment dans le rapport conflictuel qu'elle entretient avec la loi, sous-tend la pensée de Rousseau. Replacer la volonté de l'individu au centre de son système, orienté ni vers l'indépendance de l'état de nature ni vers une dépendance totale à l'égard de $1 \mathrm{la} l{ }^{2}{ }^{26}$, mais vers une autonomie morale peut sembler audacieux. Cette lecture permet cependant, pensons nous, d'interroger différemment l'œuvre de Rousseau, et notamment les notions de faible volonté, de selfdeception et de souveraineté individuelle. Elle permet également d'appréhender la question de l'unité de la pensée de Rousseau sous un jour nouveau en rejetant, après Durkheim, la

\footnotetext{
${ }^{26}$ J-F. Spitz, La liberté politique. Essai de généalogie conceptuelle, Paris!: PUF, 1995.
} 
perspective historique du second Discours et en insistant sur le statut rhétorique de son œuvre fictionnelle. Elle ne prétend pas cependant dans le cadre d'un simple article répondre à toutes les questions ni à combler tous les vides laissés par Rousseau. Le statut de la vertu, rapidement abordé à la fin de cet article, ou celui de la délibération mériteraient, par exemple, d'être plus largement développés. Mais à l'instar de la souveraineté, l'histoire des idées n'est jamais définitive et sa valeur réside essentiellement dans les questionnements et les difficultés conceptuelles qu'elle génère. 